\title{
Incidence and Risk Factors of Hospital-Acquired Pressure Ulcers Following Total Hip Arthroplasty: A Retrospective Nationwide Inpatient Sample Database Study
}

\author{
Qinfeng Yang \\ Southern Medical University \\ Jian Wang \\ Southern Medical University \\ Danping Shi \\ Southern Medical University \\ Jinlang Fu \\ Southern Medical University \\ Zhanjun Shi \\ Southern Medical University \\ Yang Zhang ( nfgjzy@126.com ) \\ Southern Medical University
}

\section{Research Article}

Keywords: Hospital-acquired pressure ulcers, Total hip arthroplasty, Nationwide Inpatient Sample

Posted Date: August 12th, 2021

DOI: https://doi.org/10.21203/rs.3.rs-764164/v1

License: (1) This work is licensed under a Creative Commons Attribution 4.0 International License. Read Full License 


\section{Abstract}

\section{Background}

The occurrence of hospital-acquired pressure ulcers (HAPUs) is disturbing and costly, leading to a variety of adverse effects. The objective of this study was to examine the incidence and risk factors of HAPUs following total hip arthroplasty (THA) using a largescale national database.

\section{Methods}

A retrospective database analysis was performed based on Nationwide Inpatient Sample (NIS) from 2005-2014. Patients who underwent THA were included. Patient demographics, hospital characteristics, length of stay (LOS), total charges during hospitalization, in-hospital mortality, preoperative comorbidities, and perioperative complications were assessed.

\section{Results}

A total of 592,174 THAs were captured from the NIS database. The general incidence of HAPUs after THA was $0.05 \%$, with a fluctuating trend annually. Patients suffered from HAPUs were older, less likely through elective admission, more likely in large hospital, more usage of Medicare, and less possibly paying via Private insurance compared to the nonaffected individuals. Additionally, the occurrence of HAPUs was associated with more preoperative comorbidities, longer LOS, extra total charges, and higher in-hospital mortality. Risk factors of HAPUs included advanced age ( $\geq 75$ years), large hospital, multiple comorbidities ( $\mathrm{n} \geq 3$ ), diabetes with chronic complications, drug abuse, liver disease, fluid and electrolyte disorders, metastatic cancer, peripheral vascular disorders, psychoses, chronic renal failure, peptic ulcer disease, and weight loss. Besides, HAPUs were associated with inflammatory arthritis and femoral neck fracture (compared with primary/secondary osteoarthritis), frailty/senility, osteoporosis, acute renal failure, pneumonia, postoperative delirium, urinary tract infection, deep vein thrombosis, sepsis/septicemia, wound dehiscence/non-healing surgical wound, periprosthetic joint infection, and mechanical prosthesis-related complications. Both elective admission and Private insurance were detected as protective factors.

\section{Conclusions}

It is beneficial to study the risk factors of HAPUs after THA to ensure the preventive management and optimize consequences although a really low incidence was identified.

\section{Background}

Total hip arthroplasty (THA) has been proven to be one of the most successful and effective procedures for alleviating pain, restoring function, and improving quality of life in patients with severe hip diseases or injuries [1]. Currently, about 400,000 THAs are performed each year in the United States, which is expected to increase up to 572,000 by 2030 [2]. However, postoperative complications may occur.

Pressure ulcers (PUs) are common complications to affect bedridden or frail hospitalized patients globally, posing substantial burden on the patients and the healthcare system [3-7]. The elderly are particularly vulnerable [5,7-9]. PUs are described as localized injuries to the skin or underlying tissue, usually over a bony prominence such as sacrum, ischial tuberosity, greater trochanter, heel, and lateral malleolus, as a result of pressure associated or pressure in combination with shear, with four stage by the depth of tissue damage (stage 1 to stage 4) [7, 9-13]. Hospital-acquired PUs (HAPUs) have constituted heavy burden on healthcare resources because they extend length of stay (LOS) and increase medical costs $[3,4,7,8,13,14]$. It is reported that the average cost of treating PUs of per individual ranges from $\$ 37,800$ to more than $\$ 127,000$ during hospitalization $[3,15,16]$. About 3 million people in the United States suffer from PUs and the overall annual cost is estimated to be exceeding $\$ 11$ billion $[7,11,14,17]$. Furthermore, HAPUs have an adverse impact on patients, family members, and health care practitioners, as these disturbing complications have been shown to be associated with greater odds of readmissions, more painful, poor function, impaired social and psychological well-being, lower quality of life for both patients and their caregivers, and even higher mortality $[3-8,10,11,13-19]$.

The reported incidence of PUs ranges greatly from $0.28-29.5 \%$, which varies for many reasons, such as patient population, different care settings, receiving operation or not, category of specific procedure, and follow-up period $[3,7-9,11,14-17,19,20]$. In order to 
optimize postoperative outcomes and prevent the occurrence of early PUs with appropriate targeting strategies, it is critical to identify preoperatively whether patients are at high risk of developing these complications $[3,4,7-9,11,12,15-19]$. A series of risk factors of PUs have been reported in previous literatures, including advanced age, frailty/senility, malnutrition, low body mass index (BMI), low level of serum albumin, immobility, skin moisture, high scores of American Society of Anesthesiology (ASA) and Charlson Comorbidity Index (CCl), diabetes, anemia, vascular disease, fluid and electrolyte disorders, respiratory disease, rheumatoid arthritis, orthopaedic operations, longer duration of surgery, acute renal failure, pneumonia, urinary tract infection, deep vein thrombosis, sepsis/septicemia, prosthesis-related complications, and postoperative delirium [3, 4, 7, 8, 10-21].

However, there is no study so far discussing the incidence and risk factors of the HAPUs after THA, based on a large-scale sample. Therefore, the purpose of this study was to investigate the incidence and risk factors of HAPUs following THA, based on a national database. Given that THA is a successful procedure to restore function for patients with severe hip diseases, this study hypothesizes that HAPUs have a relatively low incidence and several risk factors to highlight patient groups that might require preoperative optimization.

\section{Methods}

\section{Data Source}

The Nationwide Inpatient Sample (NIS) database, conducted by the Healthcare Cost and Utilization Project, and sponsored by the Agency for Healthcare Research and Quality, was the data source of this study. In the United States, NIS represents the largest allpayer database of hospital admissions. NIS collects a stratified sample from more than 1,000 hospitals, of approximately $20 \%$ of the hospitalizations in the United States each year [19, 22, 23]. The information, including patient demographics (age, sex, and race), hospital characteristics (type of admission and payer, and bedsize, teaching status, location, and region of hospital), LOS, total charges, in-hospital mortality, preoperative comorbidities, and diagnostic and procedural codes from International Classification of Diseases (ninth revision) Clinical Modification (ICD-9-CM) were obtained from this database as previously described [22]. This observational study utilized deidentified publicly available data, hence it was deemed exempt [22].

\section{Data Collection}

Patients who met the following inclusion criteria were included in this study: (i) patients with available hospitalized information registered in NIS database from 2005 to 2014; (ii) patients undergoing THA with the ICD-9-CM procedural code $81.51(n=593,045)$. Patients were excluded from the study if they were: (i) less than 18 years of age; (ii) lacked data of age $(n=871)$. According to the occurrence of HAPUs, the captured cases were divided into two groups. HAPUs were defined based on previous studies by ICD-9-CM diagnostic codes, relying on different stages and predilection sites of these complications

(707.0/707.00/707.01/707.02/707.03/707.04/707.05/707.06/707.07/707.09/707.2/707.20/707.21/707.22/707.23/707.24/707.25) $[7,19]$.

Patient demographics, hospital characteristics, preoperative comorbidities, outcome measures (LOS, total charges, and in-hospital mortality) were evaluated. The NIS database provides 29 inherent preoperative comorbidities that do not require ICD-9-CM diagnostic codes to obtain, because these inherent comorbidities have their respective variable names (Table 1). A modified Elixhauser Comorbidity Index $(\mathrm{ECl})$ including these 29 inherent preoperative comorbidities was utilized to represent the number of comorbidities [24]. Nevertheless, these 29 preoperative comorbidities still did not meet the requirements of this study, thus other additional comorbidities were defined and included at the author's discretion based on prior studies on the risk factors of PUs [11, 19, 22]. Perioperative complications before discharge were searched from the database by ICD-9-CM diagnostic code (Supplementary information file). According to prior studies, medical complications were defined as acute renal failure, acute myocardial infarction, pneumonia, pulmonary embolism, stroke, postoperative delirium, urinary tract infection, deep vein thrombosis, sepsis/septicemia, postoperative shock, and blood transfusion. Surgical complications included wound dehiscence/non-healing surgical wound, hemorrhage/seroma/hematoma, nerve injury, periprosthetic joint infection, and mechanical prosthesis-related complications [22, 25, 26]. 
Table 1

Variables Entered into the Logistic Regression Analysis

\begin{tabular}{|ll|}
\hline $\begin{array}{l}\text { Variables } \\
\text { Categories }\end{array}$ & Specific Variables \\
\hline $\begin{array}{l}\text { Patient } \\
\text { demographics }\end{array}$ & $\begin{array}{l}\text { Age ( } \leq 74 \text { years, and } \geq 75 \text { years), sex (male and female), race (White, Black, Hispanic, Asian or Pacific Islander, } \\
\text { Native American and Other) }\end{array}$ \\
$\begin{array}{l}\text { Hospital } \\
\text { characteristics }\end{array}$ & $\begin{array}{l}\text { Type of admission (non-elective, elective), bedsize of hospital (small, medium, large), teaching status of } \\
\text { hospital (nonteaching, teaching), location of hospital (rural, urban), region of hospital (Northeast, Midwest or } \\
\text { North Central, South, West), type of payer (Medicare, Medicaid, Private insurance, Self-pay, No charge, Other) }\end{array}$ \\
\hline Comorbidities & $\begin{array}{l}\text { Number of comorbidities, AlDS, alcohol abuse, deficiency anemia, rheumatoid diseases, chronic blood loss } \\
\text { anemia, congestive heart failure, chronic pulmonary disease, coagulopathy, depression, diabetes } \\
\text { (uncomplicated), diabetes (with chronic complications), drug abuse, hypertension, hypothyroidism, liver } \\
\text { disease, lymphoma, fluid and electrolyte disorders, metastatic cancer, neurological disorders, obesity, paralysis, } \\
\text { peripheral vascular disorders, psychoses, pulmonary circulation disorders, chronic renal failure, solid tumor } \\
\text { without metastasis, peptic ulcer disease, valvular disease, weight loss }\end{array}$ \\
\hline
\end{tabular}

\section{Data Analysis}

All the statistical analyses were performed using the statistical software, R version 3.5.3 (The R Foundation Inc, Auckland, New Zealand). Significant differences between two groups were determined by Wilcoxon rank test for continuous data and chi-square test for categorical data. To investigate independent risk factors of HAPUs, multivariate logistic regression with the stepwise method was conducted as previously reported [22, 23]. All variables, including demographics, hospital characteristics, and comorbidities that were provided by the NIS were entered into the regression analysis simultaneously (Table 1). Univariate and multivariate logistic regression models were constructed to evaluate the association of additional comorbidities or perioperative complications with HAPUs [22]. Statistical significance was defined by an alpha value of $P<0.05$.

\section{Results}

\section{Incidence of HAPUs in Patients Undergoing THA}

A total of 592,174 THAs were identified in the NIS database from 2005 to 2014 . Overall, there were 311 cases of HAPUs with an incidence of $0.05 \%$ (Table 2 , Table S). It was observed that the annual incidence of HAPUs following THA fluctuated during this decade (Fig. 1). 
Table 2

Patient Characteristics and Outcomes of HAPUs after THA (2005-2014)

\begin{tabular}{|c|c|c|c|}
\hline Parameter & No HAPUs & HAPUs & $P$ \\
\hline Total $(\mathrm{n}=$ count $)$ & 591,863 & 311 & - \\
\hline Total incidence (\%) & 0.05 & & \\
\hline Age (median, years) & $66(57-74)$ & $70(59-79)$ & $<0.0001$ \\
\hline \multicolumn{4}{|l|}{ Age group (\%) } \\
\hline $18-44$ & 5.07 & 4.50 & \multirow[t]{4}{*}{$<0.0001$} \\
\hline $45-64$ & 41.13 & 33.76 & \\
\hline $65-74$ & 28.97 & 22.19 & \\
\hline$\geq 75$ & 24.83 & 39.55 & \\
\hline Sex (\% female) & 56.07 & 52.09 & 0.18 \\
\hline \multicolumn{4}{|l|}{ Race (\%) } \\
\hline White & 86.47 & 83.39 & \multirow[t]{6}{*}{0.06} \\
\hline Black & 7.07 & 9.59 & \\
\hline Hispanic & 3.22 & 4.06 & \\
\hline Asian or Pacific Islander & 0.91 & 1.11 & \\
\hline Native American & 0.33 & 1.11 & \\
\hline Other & 2.00 & 0.74 & \\
\hline Type of admission (\% elective) & 91.17 & 65.27 & $<0.0001$ \\
\hline \multicolumn{4}{|l|}{ Bedsize of hospital (\%) } \\
\hline Small & 18.58 & 12.86 & \multirow[t]{3}{*}{0.02} \\
\hline Medium & 25.17 & 24.76 & \\
\hline Large & 56.25 & 62.38 & \\
\hline Teaching status of hospital (\% teaching) & 48.63 & 50.48 & 0.55 \\
\hline Location of hospital (\% urban) & 89.92 & 90.03 & 1.00 \\
\hline \multicolumn{4}{|l|}{ Region of hospital (\%) } \\
\hline Northeast & 19.78 & 18.33 & \multirow[t]{4}{*}{0.57} \\
\hline Midwest or North Central & 26.13 & 24.44 & \\
\hline South & 33.24 & 36.98 & \\
\hline West & 20.85 & 20.26 & \\
\hline ECl (median, n) & $2(1-3)$ & $3(2-4)$ & $<0.0001$ \\
\hline \multicolumn{4}{|l|}{ Comorbidities (\%) } \\
\hline 0 & 18.07 & 6.75 & \multirow[t]{3}{*}{$<0.0001$} \\
\hline 1 & 29.28 & 11.25 & \\
\hline 2 & 25.24 & 17.04 & \\
\hline
\end{tabular}

HAPUs: hospital-acquired pressure ulcers; THA: total hip arthroplasty; ECI: Elixhauser Comorbidity Index; LOS: length of stay. 


\begin{tabular}{|llll|}
\hline Parameter & No HAPUs & HAPUs & $P$ \\
\hline$\geq 3$ & 27.41 & 64.95 & $<0.0001$ \\
\hline LOS (median, days) & $3(3-4)$ & $5(3-10)$ & $<0.0001$ \\
\hline Total charges (median, \$) & $44,268(32,709-61,331)$ & $67,188(43,111-98,402)$ & 0.0005 \\
\hline Type of payer (\%) & & & \\
\hline Medicare & 52.78 & 71.75 & \\
\hline Medicaid & 3.58 & 8.12 & \\
\hline Private insurance & 40.21 & 16.88 & $<0.0001$ \\
\hline Self-pay & 0.79 & 0.65 & 0.32 \\
\hline No charge & 0.14 & 2.27 & \\
\hline Other & 2.50 & 2.89 & \\
\hline In-hospital mortality (\%) & 0.16 & & \\
\hline HAPUs: hospital-acquired pressure ulcers; THA: total hip arthroplasty; ECl: Elixhauser Comorbidity Index; LOS: length of stay. \\
\hline
\end{tabular}

\section{Patient Demographics of Two Groups}

Patients affected by HAPUs were 4 years older than those unaffected (70 years vs. 66 years, $P<0.0001$ ) (Table 2). Consistently, there was significant difference of the age distribution between the two groups, with a $14.72 \%$ higher incidence among patients older than 75 years $(39.55 \%$ vs. $24.83 \%, P<0.0001)$, while a relatively lower incidence among patients aged $18-74$ years $(60.45 \%$ vs. $75.17 \%, P$ $<0.0001)$ (Table 2). However, no significant difference was detected in gender $(P=0.18)$ or races $(P=0.06)$ between two cohorts at our defined level (Table 2).

\section{Hospital Characteristics of Two Groups}

As expected, patients suffered from HAPUs after THA were $25.90 \%$ less likely through elective admission compared to those without these complications $(65.27 \%$ vs. $91.17 \%, P<0.0001)$ (Table 2$)$. Additionally, HAPUs tended to occur in large hospital $(62.38 \%$ vs. $56.25 \%, P=0.02)$ (Table 2). Nevertheless, There was no significant difference of the hospital teaching status $(P=0.55)$, hospital bedsize $(P=1.00)$ or hospital region $(P=0.57)$ between two groups (Table 2$)$.

\section{Adverse Outcomes of HAPUs after THA}

Not surprisingly, patients with HAPUs demonstrated significant higher ECl scores ( 3 vs. $2, P<0.0001$ ), which as mentioned previously, represented more preoperative comorbidities. Besides, patients undergoing THA with multiple preoperative comorbidities $(n \geq 3)$ presented a $37.54 \%$ higher incidence of HAPUs (64.95\% vs. $27.41 \%, P<0.0001)$ (Table 2). As expected, in-hospital mortality of patients with HAPUs was significantly higher exceeding 18 times than those without HAPUs $(2.89 \%$ vs. $0.16 \%, P<0.0001)($ Table 2$)$.

The median LOS with the presence of HAPUs was 2 days longer ( 5 days vs. 3 days; $P<0.0001$ ) (Table 2). Therefore, HAPUs increased medical cost. It was found that there was an obvious increase of $\$ 22,920$ in total charges during hospitalization, with the occurrence of HAPUs $(\$ 67,188$ vs. $\$ 44,268, P<0.0001)$ (Table 2). Regarding to the type of payer, the Medicare was observed to occupied a $18.97 \%$ larger proportion $(71.75 \%$ vs. $52.78 \%$ ), while Private insurance took a $23.33 \%$ smaller proportion in the HAPUs group $(16.88 \%$ vs. $40.21 \%)(P<0.0001)$ (Table 2).

\section{Risk Factors of HAPUs after THA}

Logistic regression analysis was performed to investigate risk factors of HAPUs (Table 3 ), and the following indicators were identified: advanced age ( $\geq 75$ years; odds ratio $[\mathrm{OR}]=1.38 ; 95 \%$ confidence interval $[\mathrm{Cl}]=1.03-1.85 ; P=0.03)$, large hospital $(\mathrm{OR}=$ 1.53; $\mathrm{Cl}=1.05-2.23 ; P=0.03)$, multiple comorbidities $(\mathrm{n} \geq 3 ; \mathrm{OR}=2.33 ; \mathrm{Cl}=1.17-4.65 ; P=0.02)$, diabetes with chronic complications $(\mathrm{OR}=1.84 ; \mathrm{Cl}=1.04-3.25 ; P=0.04)$, drug abuse $(\mathrm{OR}=3.74 ; \mathrm{Cl}=2.17-6.45 ; P<0.0001)$, liver disease $(\mathrm{OR}=2.39 ; \mathrm{Cl}=1.40-4.10 ; P=$ $0.002)$, fluid and electrolyte disorders $(\mathrm{OR}=1.72 ; \mathrm{Cl}=1.28-2.31 ; P=0.0003)$, metastatic cancer $(\mathrm{OR}=2.57 ; \mathrm{Cl}=1.11-5.94 ; P=0.03)$, peripheral vascular disorders $(\mathrm{OR}=1.84 ; \mathrm{Cl}=1.20-2.83 ; P=0.005)$, psychoses $(\mathrm{OR}=1.69 ; \mathrm{Cl}=1.02-2.82 ; P=0.04)$, chronic renal 
failure $(\mathrm{OR}=1.52 ; \mathrm{Cl}=1.06-2.20 ; P=0.02)$, peptic ulcer disease $(\mathrm{OR}=13.36 ; \mathrm{Cl}=1.82-97.92 ; P=0.01)$, and weight loss $(\mathrm{OR}=3.03 ; \mathrm{Cl}$ $=1.87-4.90 ; P<0.0001)$. Interestingly, there were two protective factors of HAPUs including elective admission $(\mathrm{OR}=0.33 ; \mathrm{Cl}=0.25-$ $0.43 ; P<0.0001)$, and Private insurance $(\mathrm{OR}=0.53 ; \mathrm{Cl}=0.37-0.75 ; P=0.0004)$. 
Table 3

Risk Factors of HAPUs after THA (2005-2014)

\begin{tabular}{|c|c|c|c|}
\hline Variable & OR & $95 \% \mathrm{Cl}$ & $P$ \\
\hline \multicolumn{4}{|l|}{ Age (years) } \\
\hline$\leq 74$ & Ref. & - & - \\
\hline$\geq 75$ & 1.38 & $1.03-1.85$ & 0.03 \\
\hline Elective admission & 0.33 & $0.25-0.43$ & $<0.0001$ \\
\hline \multicolumn{4}{|l|}{ Bedsize of hospital } \\
\hline Small & Ref. & - & - \\
\hline Medium & 1.40 & $0.92-2.12$ & 0.11 \\
\hline Large & 1.53 & $1.05-2.23$ & 0.03 \\
\hline \multicolumn{4}{|l|}{ Type of payer } \\
\hline Medicare & Ref. & - & - \\
\hline Medicaid & 1.26 & $0.76-2.07$ & 0.37 \\
\hline Private insurance & 0.53 & $0.37-0.75$ & 0.0004 \\
\hline Self-pay & 0.70 & $0.17-2.87$ & 0.62 \\
\hline No charge & 1.91 & $0.26-13.85$ & 0.52 \\
\hline Other & 0.87 & $0.38-2.00$ & 0.75 \\
\hline \multicolumn{4}{|l|}{ Comorbidities } \\
\hline 0 & Ref. & - & - \\
\hline 1 & 0.85 & $0.46-1.58$ & 0.60 \\
\hline 2 & 1.26 & $0.68-2.34$ & 0.47 \\
\hline$\geq 3$ & 2.33 & $1.17-4.65$ & 0.02 \\
\hline Diabetes with chronic complications & 1.84 & $1.04-3.25$ & 0.04 \\
\hline Drug abuse & 3.74 & $2.17-6.45$ & $<0.0001$ \\
\hline Liver disease & 2.39 & $1.40-4.10$ & 0.002 \\
\hline Fluid and electrolyte disorders & 1.72 & $1.28-2.31$ & 0.0003 \\
\hline Metastatic cancer & 2.57 & $1.11-5.94$ & 0.03 \\
\hline Peripheral vascular disorders & 1.84 & $1.20-2.83$ & 0.005 \\
\hline Psychoses & 1.69 & $1.02-2.82$ & 0.04 \\
\hline Chronic renal failure & 1.52 & $1.06-2.20$ & 0.02 \\
\hline Peptic ulcer disease & 13.36 & $1.82-97.92$ & 0.01 \\
\hline Weight loss & 3.03 & $1.87-4.90$ & $<0.0001$ \\
\hline \multicolumn{4}{|c|}{ HAPUs: hospital-acquired pressure ulcers; THA: total hip arthroplasty; OR: odds ratio; Cl: confidence interval. } \\
\hline Only significant comorbidities are pre & & & \\
\hline
\end{tabular}

Additional Comorbidities Associated with HAPUs after THA 
With regard to the indications for THA, patients diagnosed with primary/secondary osteoarthritis were less likely to experience HAPUs (76.28\% vs. $95.70 \%, P=0.0005)$ (Table 4). However, those diagnosed with inflammatory arthritis $(3.16 \%$ vs. $0.85 \%, P=0.0005)$ or femoral neck fracture (FNF) $(20.55 \%$ vs. $3.45 \%, P=0.0005)$ undergoing THA were more likely to suffer from HAPUs $(P=0.0005)$ (Table 4). Furthermore, HAPUs after THA tended to occur in patients with comorbidities of Parkinson disease $(1.93 \%$ vs. $0.47 \%, P=$ $0.004)$, dementia ( $3.22 \%$ vs. $0.87 \%, P=0.0005)$, frailty/senility $(2.57 \%$ vs. $0.36 \%, P<0.0001)$, or osteoporosis $(12.86 \%$ vs. $6.06 \%, P<$ 0.0001 ) (Table 4). In multivariate analysis, inflammatory arthritis $(\mathrm{OR}=4.20 ; \mathrm{Cl}=2.06-8.54 ; P=0.0001)$ and $\mathrm{FNF}(\mathrm{OR}=6.32 ; \mathrm{Cl}=$ 4.56-8.76; $P<0.0001)$ compared with primary/secondary osteoarthritis, frailty/senility $(\mathrm{OR}=4.20 ; \mathrm{Cl}=1.85-9.55 ; P=0.0006)$, and osteoporosis $(\mathrm{OR}=1.87 ; \mathrm{Cl}=2.06-8.54 ; P=0.0008)$ were independent risk factors for HAPUs after THA (Table 4).

Table 4

Additional Comorbidities Associated with HAPUs after THA (2005-2014)

\begin{tabular}{|llllllc|}
\hline Comorbidities & \multicolumn{2}{l}{ Univariate Analysis } & \multicolumn{3}{c|}{ Multivariate Logistic Regression } \\
\cline { 2 - 7 } & No HAPUs & HAPUs & P & OR & $95 \%$ Cl & P \\
\hline Indications for THA & & & & & \\
\hline Primary/Secondary osteoarthritis & $523,550(95.70 \%)$ & $193(76.28 \%)$ & 0.0005 & Ref. & - & - \\
\hline Inflammatory arthritis & $4,635(0.85 \%)$ & $8(3.16 \%)$ & 0.0005 & 4.20 & $2.06-8.54$ & 0.0001 \\
\hline Femoral neck fracture & $18,896(3.45 \%)$ & $52(20.55 \%)$ & 0.0005 & 6.32 & $4.56-8.76$ & $<0.0001$ \\
\hline Other comorbidities & & & & & & \\
\hline Parkinson disease & $2,807(0.47 \%)$ & $6(1.93 \%)$ & 0.004 & 2.39 & $0.97-5.86$ & 0.06 \\
\hline Dementia & $5,168(0.87 \%)$ & $10(3.22 \%)$ & 0.0005 & 1.11 & $0.51-2.42$ & 0.79 \\
\hline Epilepsy & $4,496(0.76 \%)$ & $5(1.61 \%)$ & 0.09 & 1.57 & $0.58-4.23$ & 0.38 \\
\hline Frailty/Senility & $2,120(0.36 \%)$ & $8(2.57 \%)$ & $<0.0001$ & 4.20 & $1.85-9.55$ & 0.0006 \\
\hline Smoking & $121,668(20.56 \%)$ & $59(18.97 \%)$ & 0.53 & 1.00 & $0.73-1.36$ & 0.98 \\
\hline Osteoporosis & $35,859(6.06 \%)$ & $40(12.86 \%)$ & $<0.0001$ & 1.87 & $1.29-2.69$ & 0.0008 \\
\hline Chronic osteomyelitis & $192(0.03 \%)$ & $1(0.32 \%)$ & 0.10 & 0.0001 & $0.00-191.08$ & 0.97 \\
\hline HAPUs: hospital-acquired pressure ulcers; THA: total hip arthroplasty; OR: odds ratio; Cl: confidence interval. & \\
\hline
\end{tabular}

Perioperative Complications Associated with HAPUs after THA

Univariate analysis presented that patients undergoing THA with the occurrence of HAPUs were more likely to have either medical perioperative complications $(62.70 \%$ vs. $26.84 \%, P<0.0001)$ during hospitalization including acute renal failure $(14.79 \%$ vs. $1.91 \%, P$ $<0.0001)$, acute myocardial infarction ( $1.93 \%$ vs. $0.73 \%, P=0.03)$, pneumonia $(6.43 \%$ vs. $0.59 \%, P<0.0001)$, postoperative delirium (4.50\% vs. $0.96 \%, P<0.0001)$, urinary tract infection (17.68\% vs. $6.06 \%, P<0.0001)$, deep vein thrombosis $(2.25 \%$ vs. $0.27 \%, P<$ $0.0001)$, sepsis/septicemia ( $3.86 \%$ vs. $0.17 \%, P<0.0001)$, postoperative shock $(0.64 \%$ vs. $0.04 \%, P=0.007)$, and blood transfusion (43.41\% vs. $21.97 \%, P<0.0001)$, or surgical perioperative complications $(14.79 \%$ vs. $2.75 \%, P<0.0001)$ during hospitalization including wound dehiscence/non-healing surgical wound ( $1.29 \%$ vs. $0.03 \%, P<0.0001)$, hemorrhage/seroma/hematoma (3.22\% vs. $0.91 \%, P=0.0006)$, periprosthetic joint infection ( $3.22 \%$ vs. $0.21 \%, P<0.0001)$ and mechanical prosthesis-related complications $(9.65 \%$ vs. $1.68 \%, P<0.0001)$ compared with patients without HAPUs (Table 5). Multivariate analysis showed that HAPUs following THA were independently associated with acute renal failure $(\mathrm{OR}=2.83 ; \mathrm{Cl}=1.97-4.05 ; P<0.0001)$, pneumonia $(\mathrm{OR}=3.03 ; \mathrm{Cl}=1.82-$ 5.03; $P<0.0001)$, postoperative delirium ( $\mathrm{OR}=1.76 ; \mathrm{Cl}=1.01-3.07 ; P=0.04)$, urinary tract infection $(\mathrm{OR}=2.20 ; \mathrm{Cl}=1.57-3.09 ; P<$ $0.0001)$, deep vein thrombosis $(\mathrm{OR}=2.65 ; \mathrm{Cl}=1.21-5.80 ; P=0.01)$, sepsis/septicemia $(\mathrm{OR}=2.38 ; \mathrm{Cl}=1.20-4.72 ; P=0.01)$, any medical complications $(\mathrm{OR}=2.82 ; \mathrm{Cl}=1.89-4.21 ; P<0.0001)$, wound dehiscence/non-healing surgical wound $(\mathrm{OR}=8.66 ; \mathrm{Cl}=2.81-$ 26.74; $P=0.0002)$, periprosthetic joint infection $(\mathrm{OR}=2.72 ; \mathrm{Cl}=1.16-6.37 ; P=0.02)$, and mechanical prosthesis-related complications $(\mathrm{OR}=2.59 ; \mathrm{Cl}=1.08-6.19 ; P=0.03)$ (Table 5). 
Table 5

Complications Associated with HAPUs after THA (2005-2014)

\begin{tabular}{|c|c|c|c|c|c|c|}
\hline \multirow[t]{2}{*}{ Complications } & \multicolumn{3}{|c|}{ Univariate Analysis } & \multicolumn{3}{|c|}{$\begin{array}{l}\text { Multivariate Logistic } \\
\text { Regression }\end{array}$} \\
\hline & No HAPUs & HAPUs & $\mathbf{P}$ & OR & $95 \% \mathrm{Cl}$ & $\mathbf{P}$ \\
\hline \multicolumn{7}{|l|}{ Medical complications } \\
\hline Acute renal failure & $11,280(1.91 \%)$ & $46(14.79 \%)$ & $<.0001$ & 2.83 & $1.97-4.05$ & $<0.0001$ \\
\hline Acute myocardial infarction & $4,319(0.73 \%)$ & $6(1.93 \%)$ & 0.03 & 0.90 & $0.39-2.06$ & 0.80 \\
\hline Pneumonia & $3,487(0.59 \%)$ & $20(6.43 \%)$ & $<.0001$ & 3.03 & $1.82-5.03$ & $<0.0001$ \\
\hline Pulmonary embolism & $1,161(0.20 \%)$ & $2(0.64 \%)$ & 0.13 & 0.75 & $0.18-3.17$ & 0.70 \\
\hline Stroke & $4,127(0.70 \%)$ & $2(0.64 \%)$ & 1 & 0.40 & $0.09-1.63$ & 0.20 \\
\hline Postoperative delirium & $5,689(0.96 \%)$ & $14(4.50 \%)$ & <. & 1.76 & $1.01-3.07$ & 0.04 \\
\hline Urinary tract infection & $20,351(3.44 \%)$ & $55(17.68 \%)$ & $<.0001$ & 2.20 & $1.57-3.09$ & $<0.0001$ \\
\hline Deep vein thrombosis & $1,583(0.27 \%)$ & $7(2.25 \%)$ & $<.0001$ & 2.65 & $1.21-5.80$ & 0.01 \\
\hline Sepsis/Septicemia & $985(0.17 \%)$ & $12(3.86 \%)$ & $\begin{array}{l}< \\
0.0001\end{array}$ & 2.38 & $1.20-4.72$ & 0.01 \\
\hline Postoperative shock & $241(0.04 \%)$ & $2(0.64 \%)$ & 0.007 & 2.35 & $0.55-9.95$ & 0.25 \\
\hline Blood transfusion & $\begin{array}{l}130,016 \\
(21.97 \%)\end{array}$ & $\begin{array}{l}135 \\
(43.41 \%)\end{array}$ & $<.0001$ & 0.98 & $0.69-1.38$ & 0.90 \\
\hline Any medical complication ${ }^{\mathrm{a}}$ & $\begin{array}{l}158,841 \\
(26.84 \%)\end{array}$ & $\begin{array}{l}195 \\
(62.70 \%)\end{array}$ & $<.0001$ & 2.82 & $1.89-4.21$ & $<0.0001$ \\
\hline \multicolumn{7}{|l|}{ Surgical complications } \\
\hline $\begin{array}{l}\text { Wound dehiscence/Non-healing surgical } \\
\text { wound }\end{array}$ & $152(0.03 \%)$ & $4(1.29 \%)$ & $<.0001$ & 8.66 & $\begin{array}{l}2.81- \\
26.74\end{array}$ & 0.0002 \\
\hline Hemorrhage/seroma/hematoma & $5,357(0.91 \%)$ & $10(3.22 \%)$ & 0.0006 & 1.08 & $0.45-2.59$ & 0.86 \\
\hline Nerve injury & $261(0.04 \%)$ & $1(0.32 \%)$ & 0.13 & 4.45 & $\begin{array}{l}0.56- \\
35.53\end{array}$ & 0.16 \\
\hline Periprosthetic joint infection & $1,216(0.21 \%)$ & $10(3.22 \%)$ & $<.0001$ & 2.72 & $1.16-6.37$ & 0.02 \\
\hline Mechanical prosthesis-related complications & $9,941(1.68 \%)$ & $30(9.65 \%)$ & $<.0001$ & 2.59 & $1.08-6.19$ & 0.03 \\
\hline Any surgical complication ${ }^{\mathrm{b}}$ & $16,253(2.75 \%)$ & $46(14.79 \%)$ & $\dot{0} 0001$ & 1.36 & $0.55-3.34$ & 0.51 \\
\hline \multicolumn{7}{|c|}{ HAPUs: hospital-acquired pressure ulcers; THA: total hip arthroplasty; OR: odds ratio; Cl: confidence interval. } \\
\hline
\end{tabular}

\section{Discussion}

This study provides a large-scale and health-economic analysis of HAPUs after THA. To the authors' knowledge, this is the first study to explore the incidence and risk factors of HAPUs following THA. The fluctuating annual incidence of HAPUs after THA from 2005 to 2014 indicates that the effective preventive measures of HAPUs remain imperative to actually reduce the occurrence of these 
complications, such as the use of alternative pressure mattress, frequent repositioning, nutritional support, and early surgery to restore mobility recommended by prior studies $[8,9,17-19]$ (Fig. 1). An overall incidence of $0.05 \%$ of HAPUs after THA was identified, which was greatly lower compared with previous findings (0.28-29.5\%) $[3,7-9,11,14-17,19,20]$. Apart from the aforementioned several potential reasons accounting for the differences of incidences, there is another possible explanation that THA is a successful and effective procedure to restore function of mobility for most patients with severe hip diseases or FNF, consequently avoiding the requirement of staying on bed with long period $[1,8,15]$.

It has been reported that advanced age is associated with increased risk of PUs $[4,7,11,12,15,16,18,19]$. Consistent result was found in this study. Except for the elderly with poor mobility, this may also be due to that this population is commonly inherent with natural skin aging and frail skin, including dermal and epidermal thinning, decreased epidermal turnover, and loss of dermal papillae resulting in flattening of the dermoepidermal junction. Consequently, aging skin has less resistance to shear forces and a reduced contiguous surface area between the dermis and epidermis through which nutrient and oxygen transport can occur, resulting in the development of PUs $[11,15,27]$.

Not surprisingly, patients undergoing THA via elective admission were less likely to suffer from HAPUs. A possible reason for this is that most elective cases have well healthy conditions or adequate preparations preoperatively, while emergent cases tend to be severe events such as FNF in the elderly or complicated conditions lack of careful evaluations before surgery [28]. Elective admission was found as protective factor, while advanced age and FNF (compared with primary/secondary osteoarthritis) were risk factors of HAPUs further confirmed this finding. A potential explanation accounting for large hospital as risk predictors of HAPUs is that cases in these facilities are commonly challenging with complex and multiple comorbidities [22]. Private insurance was observed to take an obvious smaller proportion in the HAPUs group as well as was detected as another protective factor. This indicates that the economic status may play a role in the development of HAPUs. Besides, previous researchers presumed that clinical staffs are aware of insurance or income differences between patients and hence consciously treat them differently [29].

As expected, multiple comorbidities $(n \geq 3$ ) were associated with increased risk of HAPUs after THA, similar to the ASA and CCI mentioned above. This is apparently understandable as higher scores of these predictors commonly mean worse healthy condition or sickness of patients before surgery, and may increase the development of postoperative complications such as HAPUs $[7,15,18]$.

The occurrence of HAPUs after THA was found to be associated with extended LOS, more total charges and higher in-hospital mortality (Table 2). Several postoperative factors including associated complications, treatment of HAPUs, pain management, ambulatory ability, and family support may lead to a prolonged $\operatorname{LOS}[7,14,16,17,30,31]$. The extra total charges are due to not only the extended hospitalization, but also the treating and nursing of costly HAPUs $[4,7,14,15,31]$. Many investigators have reported that patients experiencing PUs are associated with increased rates of mortality $[4,5,8,15,17]$.

Preoperative identification of patients at increased risk of HAPUs following THA is essential to developing targeted strategies to prevent and ameliorate these adverse events $[3,4,7-9,11,12,15-19]$. Logistic regression was applied and a series of risk factors of HAPUs after THA were identified (Table 3-5). Diabetes is a well recognized risk factor for the development of PUs [3, 8, 10-12, 14$18,19,21]$. Consistently in this study, diabetes with chronic complications was at increased risk of HAPUs following THA. One possible reason accounting for this is that diabetes with chronic complications means at ending stage of disease, commonly accompanied with peripheral neuropathy which impairs the protective sensation against pressure injury [15]. Another potential explanation may be that patients with this comorbidity have poor microvascular tissue perfusion, and poor healing capacity once a tissue is injured [15]. Patients with drug abuse, psychoses, or postoperative delirium were at increased risk of HAPUs largely in line with prior findings that a lower mental status score, disorientation, and cognitive impairment were associated with the development of PUs $[11,15]$.

Malnutrition or associated nutritional factors such as weight loss, poor intake, low BMI, and low serum albumin have been reported to be associated with the development of PUs in numerous studies [4, 5, 7, 8, 11-14, 16-19]. In this study, weight loss was also found as a risk factor. A reasonable explanation is that malnutrition is frequently accompanied by loss of fat or reduction of the connective tissue that protects the skin from damage by external pressure, leading to increased the exposure of bony prominences and weakened tissue tolerance $[4,5,11,13]$. Another possible reason is that patients with weight loss is commonly related to physical weakness, dehydration, decreased mobility, edema, and poor nutritional intake which reduces nutrient availability in the body for energy metabolism, maintenance, and repair. One or a combination of these factors possibly increases the risk of formation of PUs [13]. Metastatic cancer was determined as a risk factor of HAPUs following THA, probably because this ending stage of malignancy 
is associated with weight loss and anorexia, as well as pain that limits mobility, causing the occurrence of HAPUs [32]. Besides, cancer treatment can induce anorexia and immune compromise, both of which may confer increased risk for HAPUs and impaired wound healing [32].

Peripheral vascular disorders also has been reported frequently to be associated with PUs. [3, 7, 8, 12, 16-19, 21]. A possible reason accounting for this is that these patients commonly exist with hypoperfusion which impairs the delivery of oxygen, nutrients and removal of wastes in patients with vulnerable skin and subcutaneous tissue, and further impairs wound healing $[12,17,21]$. Despite fluid and electrolyte disorders have been found to be associated with increased risk of PUs, the reason remains unclear [14, 19]. We assume that this is likely associated with poor nutritional status which may compromise skin integrity [4]. It was found that both chronic renal failure and acute renal failure were associated with the occurrence of HAPUs in this study. Nevertheless, studies about the certain relationship between renal failure and PUs are rare $[7,16,19,21]$. It is likely attributed to metabolism or nutrition related factors. To the authors' knowledge, peptic ulcer disease and liver disease were first found as risk factors of HAPUs after THA. Interestingly, peptic ulcer disease introduced the highest risk among all the factors (Table 3). A potential explanation may be that patients with these digestive system diseases were usually accompanied by poor nutritional intake which reduces nutrient availability in the body for energy metabolism, maintenance, and repair [13].

It was observed in this study that patients with indications for THA such as inflammatory arthritis (e.g., rheumatoid arthritis) or FNF had higher odds of HAPUs compared with primary/secondary osteoarthritis. Patients with rheumatoid arthritis typically receiving the therapy of corticosteroids may cause skin fragility and hinder tissue repair, thus contributing to the risk [16, 33]. Patients with FNF undergoing THA are prone to experiencing HAPUs probably because of exposure to long periods of immobility before, during and after surgery [34]. In accordance with previous studies, it was found that frailty/senility was associated with increased risk of HAPUs $[11,18,19,35,36]$. This may be due to that the elderly who are frail have a diminished capacity to compensate effectively for external stressors and hence are vulnerable to adverse outcomes such as PUs [35, 36]. Osteoporosis was detected as risk factor of HAPUs possibly as a result of poor nutrition. Furthermore, osteoporosis is a main cause of FNF especially among the elderly, and these factors are found to be associated with HAPUs as mentioned above [37].

Several infection-related complications including pneumonia, urinary tract infection, sepsis/septicemia, and periprosthetic joint infection were found to be associated with HAPUs. Impaired inflammatory response, insufficient oxygen supplement and decreased mobility in individuals with pneumonia may predispose them to develop HAPUs [38]. Patients with urinary tract infection are prone to suffering from HAPUs likely because they are usually accompanied by urinary incontinence or moisture of local skin $[7,8,11,12,15$, 19]. It has been reported that patients with the occurrence of sepsis/septicemia are in a high frailty state that may predispose them to PUs development [15]. Although THA is a successful and effective procedure to restore function of mobility for most patients with severe hip diseases or FNF, periprosthetic joint infection and mechanical prosthesis-related complications are still challenging for both surgeons and patients [1,22]. These individuals are vulnerable to experiencing HAPUs possibly as a result of limited mobility [19]. Besides, deep vein thrombosis has also been found to be associated with PUs [11, 19]. Furthermore, deep vein thrombosis, pneumonia, urinary tract infection and PUs are regarded as major immobility complications [39]. Therefore, immobility plays a critical role in developing PUs [11]. Similarly, patients with wound dehiscence/non-healing surgical wound after THA were restricted with movement may contribute to the occurrence of HAPUs [11].

The main strengths of our study include its large sample size as well as national representativeness, and the application of multivariable regression modeling to mitigate confounding. However, several limitations must be acknowledged mainly inherent to the utilization of the NIS database. First, information of each patient is only recorded during hospitalization, suggesting any complication or outcome post-discharge such as rates of readmission and long term follow-up will not be provided by this database. This limitation might result in underestimating the incidence of PUs as only early period in-hospital cases were captured [17, 19, 22, $23,25,26]$. Second, only variables recorded in the NIS database could be analyzed. There are other known risk factors associated with PUs were not available in the NIS database, such as duration of surgery, BMI, ASA, level of serum albumin, immobility, and skin moisture $[7,11,12,16,18]$. Additionally, as with any large administrative database, discrepancy or misclassification in coding and documentation may occur $[19,22,23,25]$.

\section{Conclusions}

Page 12/16 
HAPUs following THA are challenging and costly with an overall incidence of $0.05 \%$. The annual incidence of HAPUs was fluctuating from 2005 to 2014. Several risk factors of HAPUs after THA were identified in this study including advanced age ( $\geq 75$ years), large hospital, multiple comorbidities $(n \geq 3)$, diabetes with chronic complications, drug abuse, liver disease, fluid and electrolyte disorders, metastatic cancer, peripheral vascular disorders, psychoses, chronic renal failure, peptic ulcer disease, and weight loss. Furthermore, HAPUs were associated with inflammatory arthritis and FNF (compared with primary/secondary osteoarthritis), frailty/senility, osteoporosis, acute renal failure, pneumonia, postoperative delirium, urinary tract infection, deep vein thrombosis, sepsis/septicemia, wound dehiscence/non-healing surgical wound, periprosthetic joint infection, and mechanical prosthesis-related complications. Both elective admission and Private insurance were detected as protective factors. Patients with HAPUs after THA presented more preoperative comorbidities, prolonged LOS, excess total charges, and higher in-hospital mortality.

\section{Abbreviations}

\section{ASA}

American Society of Anesthesiology

BMI

Body mass index

$\mathrm{CCl}$

Charlson Comorbidity Index

$\mathrm{Cl}$

Confidence interval

$\mathrm{ECl}$

Elixhauser Comorbidity Index

FNF

Femoral neck fracture

HAPUs

Hospital-acquired pressure ulcers

ICD-9-CM

International Classification of Diseases (ninth revision) Clinical Modification

LOS

Length of stay

NIS

Nationwide Inpatient Sample

OR

Odds ratio

PJI

Periprosthetic joint infection

PRCs

Prosthesis-related complications

PUs

Pressure ulcers

THA

Total hip arthroplasty

\section{Declarations}

\section{Ethics approval and consent to participate}

Not applicable.

\section{Consent for publication}

Not applicable. 
All data generated or analysed during this study are included in this published article and its supplementary information files.

\section{Competing interests}

The authors declare that they have no competing interest.

\section{Funding}

This research did not receive any specific grant from funding agencies in the public, commercial, or not-for-profit sectors.

\section{Authors' contributions}

QY contributed to the study design, data acquisition and analysis, interpretation of results, and writing and revising the manuscript. JW and ZS contributed to the study design, interpretation of results, and reviewing the manuscript. DS and JF contributed to data acquisition, data analysis, and reviewing of the manuscript. YZ contributed to the study design, interpretation of results, and reviewing the manuscript. All authors read and approved the final manuscript.

\section{Acknowledgements}

We express our sincere gratitude to Goodwill Hessian Health Technology Co. Ltd. (100007, Beijing, China.) for providing consultation and guidance on statistical analysis in this study.

\section{References}

1. Bozic KJ, Kurtz SM, Lau E, Ong K, Vail DTP, Berry DJ. The epidemiology of revision total hip arthroplasty in the united states. J Bone Joint Surg Am. 2009;91:128-33.

2. Kurtz S, Ong K, Lau E, Mowat F, Halpern M. Projections of primary and revision hip and knee arthroplasty in the United States from 2005 to 2030. J Bone Joint Surg Am. 2007;89:780-5.

3. Rao AD, Preston AM, Strauss R, Stamm R, Zalman DC. Risk factors associated with pressure ulcer formation in critically ill cardiac surgery patients a systematic review. J Wound Ostomy Continence Nurs. 2016;43:242-7.

4. Raju D, Su X, Patrician PA, Loan LA, McCarthy MS. Exploring factors associated with pressure ulcers: A data mining approach. Int J Nurs Stud. 2015;52:102-11.

5. lizaka S, Okuwa M, Sugama J, Sanada H. The impact of malnutrition and nutrition-related factors on the development and severity of pressure ulcers in older patients receiving home care. Clin Nutr. 2010;29:47-53. doi:10.1016/j.clnu.2009.05.018.

6. Yang L, Xiao Z, An P, Yan H, Li Q. Association between pressure ulcers and the risk of postoperative infections in male adults with spinal cord injury. Br J Neurosurg. 2020;0:1-4.

7. Gardiner JC, Reed PL, Bonner JD, Haggerty DK, Hale DG. Incidence of hospital-acquired pressure ulcers - a population-based cohort study. Int Wound J. 2016;13:809-20.

8. Magny $\mathrm{E}$, Vallet $\mathrm{H}$, Cohen-Bittan J, Raux M, Meziere A, Verny M, et al. Pressure ulcers are associated with 6-month mortality in elderly patients with hip fracture managed in orthogeriatric care pathway. Arch Osteoporos. 2017;12:77.

9. Bredesen IM, Bjøro K, Gunningberg L, Hofoss D. The prevalence, prevention and multilevel variance of pressure ulcers in Norwegian hospitals: A cross-sectional study. Int J Nurs Stud. 2015;52:149-56.

10. Lopes TS, Videira LMM dos S, Saraiva DMRF, Agostinho ES, Bandarra AJF. Multicentre study of pressure ulcer point prevalence in a Portuguese region. J Tissue Viability. 2020;29:12-8.

11. Mervis JS, Phillips TJ. Pressure ulcers: Pathophysiology, epidemiology, risk factors, and presentation. J Am Acad Dermatol. 2019;81:881-90.

12. Coleman S, Gorecki C, Nelson EA, Closs SJ, Defloor T, Halfens R, et al. Patient risk factors for pressure ulcer development: Systematic review. Int J Nurs Stud. 2013;50:974-1003.

13. Banks $\mathrm{M}, \mathrm{Ph} \mathrm{D}$, Bauer $\mathrm{J}, \mathrm{Ph} \mathrm{D}$, Graves $\mathrm{N}, \mathrm{Ph} \mathrm{D}$, et al. Malnutrition and pressure ulcer risk in adults in Australian health care facilities. Nutrition. 2010;26:896-901. 
14. Dreyfus J, Gayle J, Trueman P, Delhougne G, Siddiqui A. Assessment of Risk Factors Associated With Hospital-Acquired Pressure Injuries and Impact on Health Care Utilization and Cost Outcomes in US Hospitals. Am J Med Qual. 2018;33:348-58.

15. Galivanche AR, Kebaish KJ, Adrados M, Ottesen TD, Varthi AG, Rubin LE, et al. Postoperative Pressure Ulcers after Geriatric Hip Fracture Surgery Are Predicted by Defined Preoperative Comorbidities and Postoperative Complications. J Am Acad Orthop Surg. 2020;28:342-51.

16. Geller CM, Seng SS. How to keep patients un-HAPI: Cardiac surgery and sacral pressure injuries: Invited expert opinion: Hospitalacquired pressure injuries. J Thorac Cardiovasc Surg. 2020;160:158-63.

17. Grigorian A, Sugimoto M, Joe V, Schubl S, Lekawa M, Dolich M, et al. Pressure Ulcer in Trauma Patients: A Higher Spinal Cord Injury Level Leads to Higher Risk. J Am Coll Clin Wound Spec. 2017;9:24-31.e1.

18. Aloweni F, Ang SY, Fook-Chong S, Agus N, Yong P, Goh MM, et al. A prediction tool for hospital-acquired pressure ulcers among surgical patients: Surgical pressure ulcer risk score. Int Wound J. 2019;16:164-75.

19. Fogerty MD, Abumrad NN, Nanney L, Arbogast PG, Poulose B, Barbul A. Risk factors for pressure ulcers in acute care hospitals. Wound Repair Regen. 2008;16:11-8.

20. Sardo PMG, Simões CSO, Alvarelhão JJM, Simões JFFL, Machado PAP, Amado FML, et al. Analyses of pressure ulcer incidence in inpatient setting in a Portuguese hospital. J Tissue Viability. 2016;25:209-15.

21. Lumbley JL, Ali SA, Tchokouani LS. Retrospective review of predisposing factors for intraoperative pressure ulcer development. J Clin Anesth. 2014;26:368-74.

22. Yang Q, Wang J, Xu Y, Chen Y, Lian Q, Zhang Y. Incidence and risk factors of in-hospital prosthesis-related complications following total hip arthroplasty: a retrospective Nationwide Inpatient Sample database study. Int Orthop. 2020;44:2243-52.

23. Fineberg SJ, Nandyala S V., Marquez-Lara A, Oglesby M, Patel AA, Singh K. Incidence and risk factors for postoperative delirium after lumbar spine surgery. Spine (Phila Pa 1976). 2013;38:1790-6.

24. Sayeed Z, Anoushiravani AA, Chambers MC, Gilbert TJ, Scaife SL, El-Othmani MM, et al. Comparing In-Hospital Total Joint Arthroplasty Outcomes and Resource Consumption Among Underweight and Morbidly Obese Patients. J Arthroplasty. 2016;31:2085-90.

25. Buller LT, Rosas S, Sabeh KG, Roche MW, McLawhorn AS, Barsoum WK. Hypothyroidism Increases 90-Day Complications and Costs Following Primary Total Knee Arthroplasty. J Arthroplasty. 2018;33:1003-7.

26. Newman JM, Sodhi N, Dalton SE, Khlopas A, Newman RP, Higuera CA, et al. Does Parkinson Disease Increase the Risk of Perioperative Complications After Total Hip Arthroplasty? A Nationwide Database Study. J Arthroplasty. 2018;33:S162-6.

27. Farage MA, Miller KW, Elsner P, Maibach HI. Characteristics of the Aging Skin. Adv Wound Care. 2013;2:5-10.

28. Sams JD, Milbrandt JC, Froelich JM, Rainville AD, Allan DG. Hospital Outcome After Emergent vs Elective Revision Total Hip Arthroplasty. J Arthroplasty. 2010;25:826-8.

29. Shen JJ, Cochran CR, Mazurenko O, Moseley CB, Shan G, Mukalian R, et al. Racial and insurance status disparities in patient safety indicators among hospitalized patients. Ethn Dis. 2016;26:443-52.

30. Kurtz SM, Ms ECL, Ong KL, Adler EM, Kolisek FR, Frsa MTM. Which Clinical and Patient Factors Influence the National Economic Burden of Hospital Readmissions After Total Joint Arthroplasty ? Clin Orthop Relat Res. 2017;475:2926-37.

31. Theisen S, Drabik A, Stock S. Pressure ulcers in older hospitalised patients and its impact on length of stay: A retrospective observational study. J Clin Nurs. 2012;21:380-7.

32. Levine JM, Menezes R, Namagiri S. Wounds Related to Malignancy in Postacute and Long-term Care: A Case Series. Adv Ski Wound Care. 2020;33:99-102.

33. Firth J, Waxman R, Law G, Nelson EA, Helliwell P, Siddle H, et al. The predictors of foot ulceration in patients with rheumatoid arthritis. Clin Rheumatol. 2014;33:615-21.

34. Baumgarten M, Margolis DJ, Orwig DL, Shardell MD, Hawkes WG, Langenberg P, et al. Pressure ulcers in elderly patients with hip fracture across the continuum of care. J Am Geriatr Soc. 2009;57:863-70.

35. Hubbard RE, Peel NM, Samanta M, Gray LC, Mitnitski A, Rockwood K. Frailty status at admission to hospital predicts multiple adverse outcomes. Age Ageing. 2017;46:801-6.

36. McRae PJ, Walker PJ, Peel NM, Hobson D, Parsonson F, Donovan P, et al. Frailty and Geriatric Syndromes in Vascular Surgical Ward Patients. Ann Vasc Surg. 2016;35 May:9-18.

Page 15/16 
37. Han TS, Yeong K, Lisk R, Fluck D, Fry CH. Prevalence and consequences of malnutrition and malnourishment in older individuals admitted to hospital with a hip fracture. Eur J Clin Nutr. 2020.

38. Krishnan S, Karg PE, Boninger ML, Brienza DM. Association between presence of pneumonia and pressure ulcer formation following traumatic spinal cord injury. J Spinal Cord Med. 2017;40:415-22.

39. Li Z, Zhou X, Cao J, Li Z, Wan X, Li J, et al. Nurses' knowledge and attitudes regarding major immobility complications among bedridden patients: A prospective multicentre study. J Clin Nurs. 2018;27:1969-80.

\section{Figures}

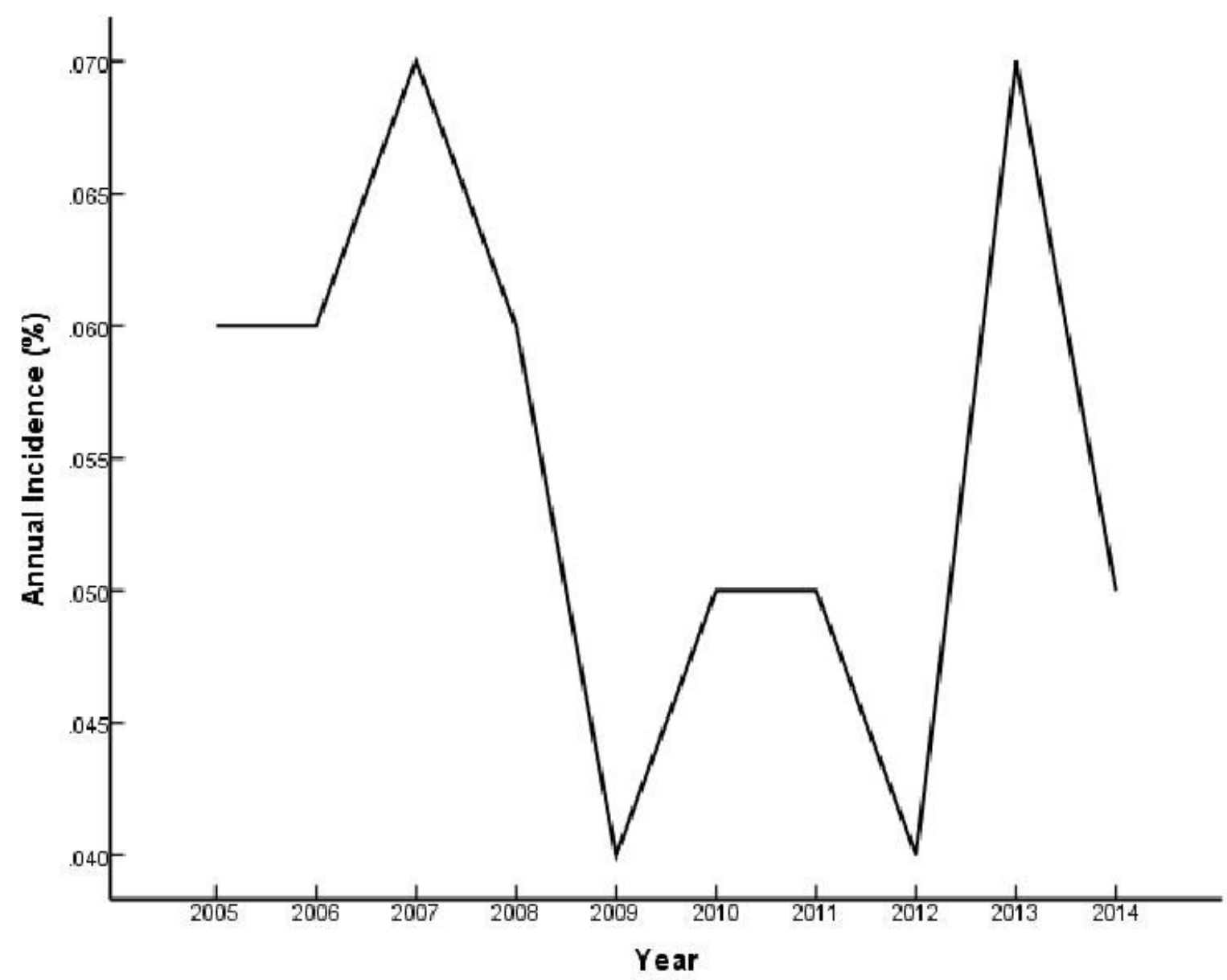

\section{Figure 1}

Annual incidence of hospital-acquired pressure ulcers (HAPUs) following total hip arthroplasty (THA) from 2005 to 2014 . This shows that the incidence of HAPUs after THA fluctuated during this decade.

\section{Supplementary Files}

This is a list of supplementary files associated with this preprint. Click to download.

- Supplementaryinformationfile.doc 